\title{
Phyllodes Tumor: A Rare Case with Cervical Vertebral Metastasis
}

\author{
Shankar Ayyappan Kutty ${ }^{1}$ Lilly Rajeevan ${ }^{2}$ \\ ${ }^{1}$ NMC Specialty Hospital, Abu Dhabi, United Arab Emirates \\ ${ }^{2}$ Department of Pathology, Malabar Institute of Medical Sciences, \\ Kozhikode, Kerala, India \\ Address for correspondence Dr. Shankar Ayyappan Kutty, MCh, NMC \\ Specialty Hospital, PO Box 6222, Abu Dhabi, United Arab Emirates \\ (e-mail: shankar.ayyappan@nmc.ae).
}

Indian J Neurosurg 2015;4:35-37.

\begin{abstract}
Keywords

- cystosarcoma phyllodes

- cervical spine

- metastasis

- vertebrae

Background Phyllodes tumor or cystosarcoma phyllodes is a rare tumor that accounts for less than $1 \%$ of breast lesions. Incidence of these lesions metastasizing to distant sites is very low and only a few cases of metastases to central nervous system have been reported. The management of a case with cervical spine metastasis is described and current literature has been reviewed.

Case Description A 32-year-old woman who underwent excision of cystosarcoma phyllodes of the right breast presented with neck pain. Evaluation showed destruction of the C3-C4 vertebrae. Anterior decompression with anterior and posterior fusion was done, followed by radiotherapy.

Conclusion Spinal metastasis from cystosarcoma phyllodes is extremely rare, but should be kept in mind when a patient presents with symptoms suggestive of a spinal problem. Surgical management of these tumors may enhance the quality of life of these patients.
\end{abstract}

\section{Introduction}

Phyllodes tumor is a benign fibroepithelial lesion of the breast, which can become malignant in a minority of cases. Local recurrence and spread is common, but distant metastasis is rare. Metastases, however, have been known to occur to most organs, including the brain and thoracolumbar spine. ${ }^{1}$

\section{Case Report}

A 32-year-old lady, who had undergone surgery for cystosarcoma phyllodes involving the right breast 1 year earlier, presented with severe neck pain of 2-month duration. There was no radiation of pain to the upper limbs and no symptoms suggestive of motor or sensory deficits. Micturition was normal.

She was fully conscious and oriented. General examination was unremarkable. There were no focal neurological deficits. Neck movements were restricted, and there was tenderness of the cervical spine.
X-ray of the cervical spine showed the collapse of C3 vertebra, and MRI of the cervical spine ( - Fig. 1) revealed a destructive lesion involving the $\mathrm{C} 3$ and $\mathrm{C} 4$ vertebral bodies, causing total collapse of $\mathrm{C} 3$ body and a large prevertebral soft tissue component at the level of the epiglottis. A small epidural component was present at the same level causing thecal compression. There was no compression of the cord. The pedicles were involved bilaterally at C3 and C4 levels.

She underwent surgical decompression of the soft tissue component. The lesion was found to be a compressible, rubbery, vascular mass in the prevertebral plane at C3-4 level with extension into the vertebral bodies, causing total destruction of $\mathrm{C} 3$ body and destruction of the upper part of C4 body. The lesion was extending under the C4 body in the extradural space. At the end of the surgery, an expandable cage (Xpand, Globus Medical Audubon, Pennsylvania, United States) filled with bone graft was placed in the corpectomy defect anteriorly. Posteriorly pedicle screws were placed on C5 and lamina hooks on C2, and fusion was completed with rods and bone graft. published online March 20, 2015
DOI http://dx.doi.org/ 10.1055/s-0035-1549062. ISSN 2277-954X. (c) 2015 Neurological Surgeons' Society of India

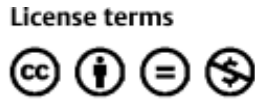




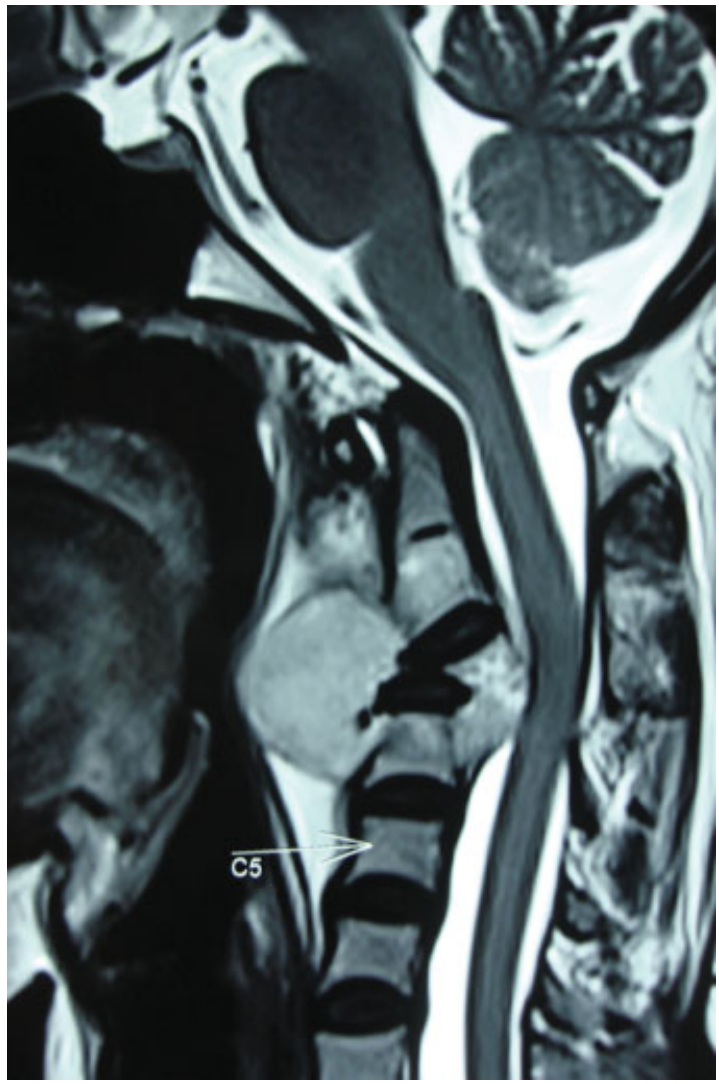

Fig. 1 MRI (T2-weighted) showing total destruction of C3 body with preserved disc spaces and a large prevertebral soft tissue lesion.

The biopsy was reported as being consistent with a metastatic high-grade phyllodes tumor (-Fig. 2-4). Postoperatively she had complete relief of pain. After the wound had healed, she was referred to the oncologist for radiation and chemotherapy to control the growth of the lesion. A repeat imaging of the cervical spine done 6 months later showed evidence of local recurrence, but there was no spinal compression. A CT scan of the thorax done at the same time revealed multiple lesions involving both lungs (-Fig. 5) and hence she was started on chemotherapy. Two

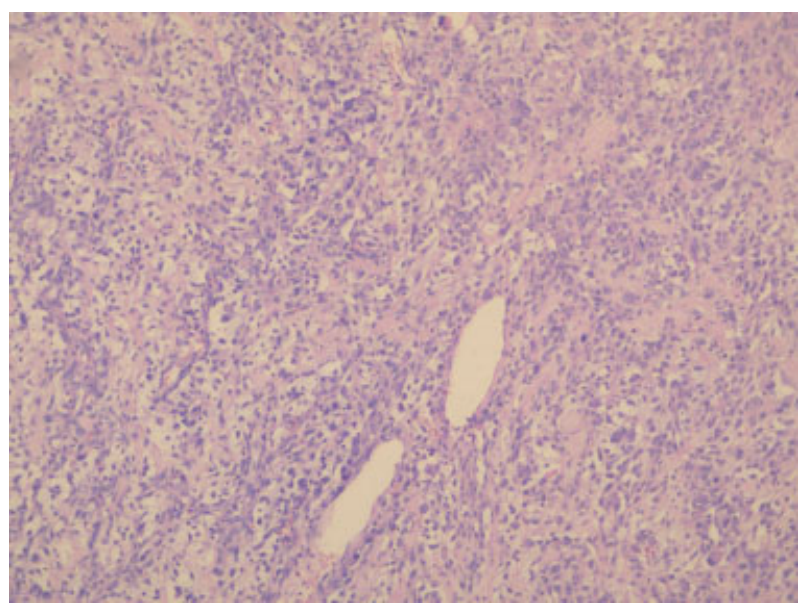

Fig. 2 Spindly tumor cells with oval hyperchromatic nuclei arranged in sheets (hematoxylin and eosin).

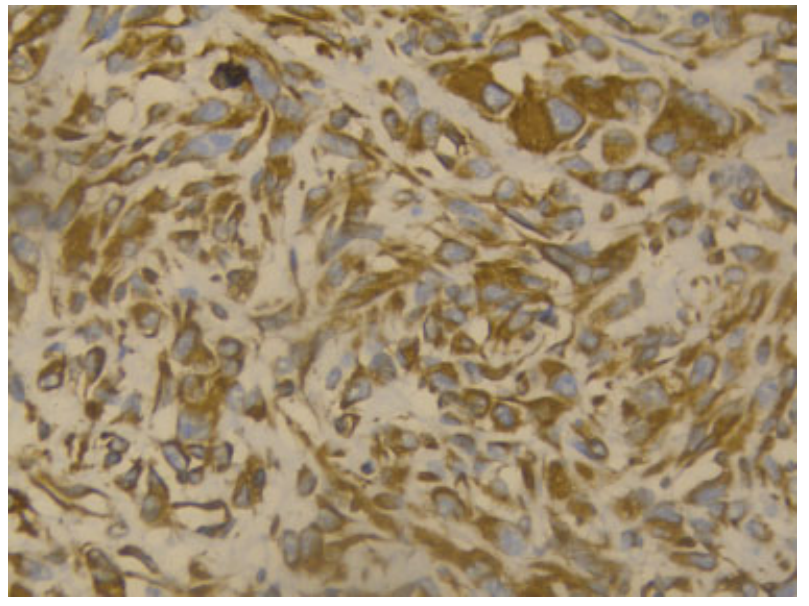

Fig. 3 Spindly cells exhibiting vimentin positivity (immunohistochemistry).

years after the surgery, she died following an episode of uncontrolled hemoptysis. Till the time of her death, she was ambulant and active, but had pain in her shoulders and neck.

\section{Discussion}

Cystosarcoma phyllodes, also known as phyllodes tumors, are rare tumors that involve breast. First reported by Johannes Muller in 1838, they make up less than $1 \%$ of all breast tumors. ${ }^{1}$ The majority of these lesions behave in a completely benign manner, leaving local recurrence as the only real concern. However, up to $30 \%$ of these lesions show histopathological features of malignancy, though the clinical course may not correlate with the histopathological grading. $^{2}$

These tumors can occur at any age, but present most commonly in the fifth decade of life. ${ }^{3}$ The mean age of presentation for recurrent lesions is 52 years. The time to metastasis varies from 3.5 months to 14 years from initial diagnosis in various studies. ${ }^{4,5}$

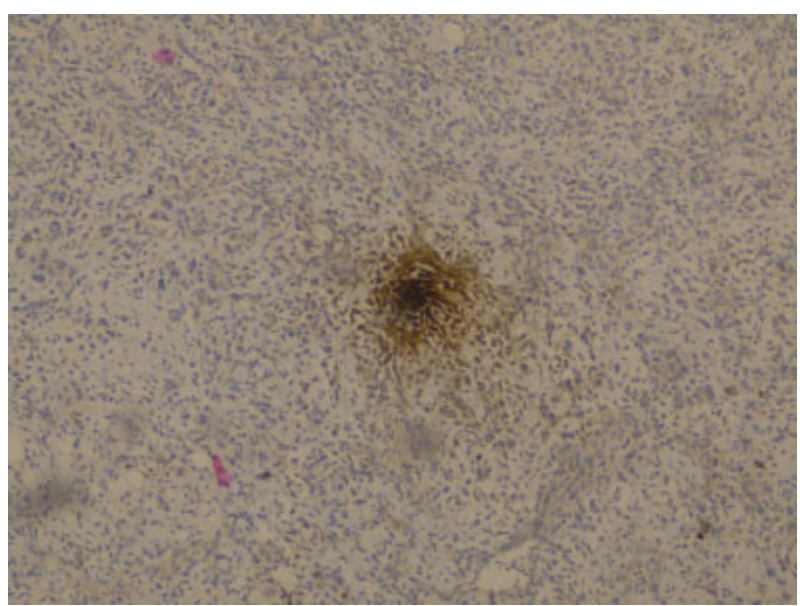

Fig. 4 Spindly cells exhibiting cytokeratin positivity (immunohistochemistry). 


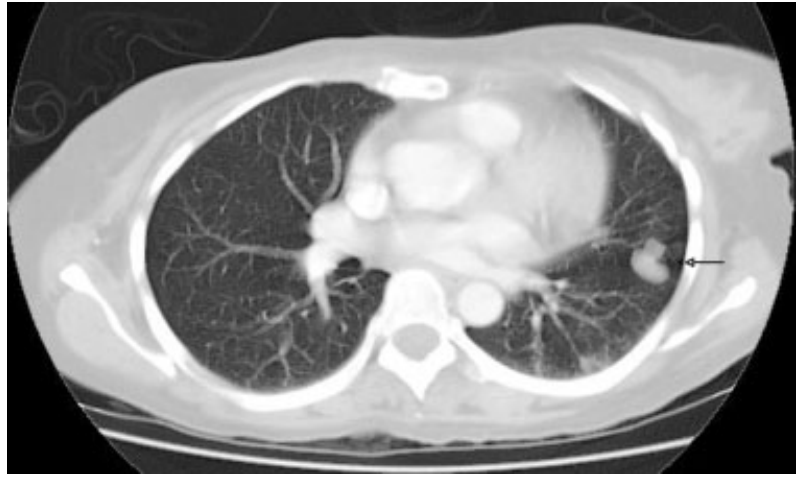

Fig. 5 Axial section of the CT scan of the thorax (contrast) showing an enhancing nodule on the left side (black arrow).

Histopathologically, they are characterized by a combination of hypercellular stroma and cleft-like or cystic spaces lined by epithelium, into which the stroma classically projects in a leaf-like fashion. This appearance is in part responsible for the name, "phyllos" meaning "leaves." Stromal cellularity and infiltrating tumor margins are predictors of local recurrence. ${ }^{6}$ About $15 \%$ of patients with phyllodes tumor develop local recurrence, while 5 to $10 \%$ develop metastases. ${ }^{2}$ This figure goes up to $20 \%$ for patients with malignant phyllodes tumors. The hematogenous route is usually responsible for the metastatic disease, and lymphatic spread is rare. ${ }^{6}$ Most distant metastases may develop without evidence of local recurrence. ${ }^{7}$

Central nervous system (CNS) metastases are uncommon, and most cases reported in the English literature show a spread to the brain, with dural-based lesions mimicking meningiomas being a common presentation. ${ }^{8}$ Metastases to the sacrum and thoracolumbar spine have also been reported, but are very rare. Patients with brain metastases have a dismal mean survival of less than a month, but those with bony metastases, including spine, have a much better prognosis with 24-month survival being reported. Our patient also survived for 2 years after initial presentation. Though these tumors are believed to be radiosensitive, the prognosis depends on the tumor load since most of the patients have multiple metastases by the time they develop CNS disease. ${ }^{9}$
We believe that this is the first case of cystosarcoma phyllodes with cervical spine metastasis to be reported. The malignant nature of the disease with multiple metastases to the lungs is also well demonstrated. The importance of spinal stabilization to safeguard the quality of life of these patients has to be stressed, since the survival for patients with spinal metastases is much longer than for those with brain metastases. ${ }^{9}$

\section{Conflict of Interest}

Nil.

\section{Sources of Support}

Nil.

\section{References}

1 Rosenfeld JC, DeLaurentis DA, Lerner H. Cystosarcoma phyllodes. Diagnosis and management. Cancer Clin Trials 1981;4(2): 187-193

2 Telli ML, Horst KC, Guardino AE, Dirbas FM, Carlson RW. Phyllodes tumors of the breast: natural history, diagnosis, and treatment. J Natl Compr Canc Netw 2007;5(3):324-330

3 Lee BJ, Pack GT. Giant intracanalicular myxoma of the breast, socalled cystosarcoma phyllodes mammae of Johannes Muller. Ann Surg 1931;93(1):250-268

4 Dyer NH, Bridger JE, Taylor RS. Cystosarcoma phylloides. Br J Surg 1966;53(5):450-455

5 Grimes MM, Lattes R, Jaretzki A III. Cystosarcoma phyllodes. Report of an unusual case, with death due to intraneural extension to the central nervous system. Cancer 1985;56(7): 1691-1695

6 Moffat CJC, Pinder SE, Dixon AR, Elston CW, Blamey RW, Ellis IO. Phyllodes tumours of the breast: a clinicopathological review of thirty-two cases. Histopathology 1995;27(3):205-218

7 Palmer ML, De Risi DC, Pelikan A, et al. Treatment options and recurrence potential for cystosarcoma phyllodes. Surg Gynecol Obstet 1990;170(3):193-196

8 Rhodes RH, Frankel KA, Davis RL, Tatter D. Metastatic cystosarcoma phyllodes. A report of 2 cases presenting with neurological symptoms. Cancer 1978;41(3):1179-1187

9 Hlavin ML, Kaminski HJ, Cohen M, Abdul-Karim FW, Ganz E. Central nervous system complications of cystosarcoma phyllodes. Cancer 1993;72(1):126-130 